\title{
SEVERAL THEOREMS ON BOUNDEDNESS AND EQUICONTINUITY ${ }^{1}$
}

\author{
J. D. STEIN, JR.
}

ABSTRACT. This paper presents several results concerning equicontinuity of a pointwise-bounded family of linear transformations on a Banach space. The first is the following generalization of the Banach-Steinhaus Theorem: Let $\left\{T_{\alpha} \mid \alpha \in A\right\}$ be a pointwisebounded family of linear transformations from a Banach space $X$ to a normed linear space $Y$, and assume that, for each $\alpha \in A, T_{\alpha}$ is continuous on a closed subspace $S_{\alpha}$ of $X$. Then $\exists \alpha_{1}, \cdots, \alpha_{n} \in A$ such that the family is equicontinuous on $\bigcap_{k=1}^{n} S_{\alpha_{k}}$. The second theorem deals with a pointwise-bounded family of linear transformations from a Banach space $X$ to a normed linear space with a continuous bilinear mapping into another normed linear space. The others deal with homomorphisms of Banach algebras.

The purpose of this paper is to present several theorems illustrating a method by which equicontinuity can be deduced from pointwiseboundedness, for families of linear transformations whose domain is a Banach space.

The first theorem simultaneously generalizes the classical BanachSteinhaus Theorem and a Uniform Boundedness Theorem of Pták [4].

THEOREM 1. Let $\mathcal{F}=\left\{T_{\alpha} \mid \alpha \in A\right\}$ be a pointwise-bounded family of linear transformations from a Banach space $X$ to a normed space $Y$. Let $S_{\alpha}$ be a closed subspace of $X$ such that $T_{\alpha} \mid S_{\alpha}$ is continuous, $\forall \alpha \in A$. Then $\exists \alpha_{1}, \cdots, \alpha_{n} \in A$ such that $\mathcal{F}$ is equicontinuous on $\bigcap_{k=1}^{n} S_{\alpha_{k}}$.

Proof. For $x \in X$ let $M(x)=\sup \left\{\left\|T_{\alpha} x\right\| \mid \alpha \in A\right\}$, and let $r(\alpha)$ denote the norm of $T_{\alpha}$ restricted to $S_{\alpha}$. Assume the theorem false. Then, given any finite set of pairs $\left(S_{1}, T_{1}\right), \cdots,\left(S_{p}, T_{p}\right)$ with $T_{k} \in \mathcal{F}$ nonzero and continuous on the closed subspace $S_{k}, 1 \leqq k \leqq p$, and given any constant $M>0$, there is a $T \in \mathcal{F}$ and $x \in \bigcap_{k=1}^{p} S_{k}$ with $\|x\| \leqq 1,\|T x\|>M$, and $\left\|T_{k} x\right\| \leqq 1$ for $1 \leqq k \leqq p$.

Since $\mathcal{F}$ is not equicontinuous on any $S_{\alpha}$, choose $T_{1} \in \mathcal{F}$ and $x_{1} \in X$ with $\left\|x_{1}\right\| \leqq 1,\left\|T_{1} x_{1}\right\|>4$. Inductively, choose sequences

Presented to the Society, March 27, 1970 under the title Boundedness, orthogonality, and equicontinuity; received by the editors February 11, 1970.

AMS 1969 subject classifications. Primary 4610.

Key words and phrases. Functional analysis, Banach spaces.

2 The preparation of this paper was supported in part by NSF Grant GP-11475. 
$\left\{x_{n} \mid n=1,2, \cdots\right\} \subseteq X, \quad\left\{T_{n} \mid n=1,2, \cdots\right\} \subseteq F$ with $\left\|x_{n}\right\| \leqq 1$, $x_{n} \in \bigcap_{k=1}^{n-1} S_{k}$ for $n \geqq 2$, where $S_{k}$ is the closed subspace on which $T_{k}$ is continuous, and $\left\|T_{k} x_{n}\right\| \leqq 1$ for $1 \leqq k \leqq n-1, \quad\left\|T_{n} x_{n}\right\|>$ $2^{n}\left(n+1+\sum_{j=1}^{n-1} 2^{-j} M\left(x_{j}\right)\right)$. Let $x=\sum_{k=1}^{\infty} 2^{-k} x_{k}$, by completeness, $x \in X$. For $n \geqq 2,\left\|T_{n} x\right\|=\left\|2^{-n} T_{n} x_{n}+\sum_{k=1}^{n-1} 2^{-k} T_{n} x_{k}+T_{n}\left(\sum_{k=n+1}^{\infty} 2^{-k} x_{k}\right)\right\|$. But $k \geqq n+1 \Rightarrow x_{k} \in S_{n}$, and since $S_{n}$ is closed and $T_{n} \mid S_{n}$ is continuous, $T_{n}\left(\sum_{k=n+1}^{\infty} 2^{-k} x_{k}\right)=\sum_{k=n+1}^{\infty} 2^{-k} T_{n} x_{k}$, and so

$$
\begin{aligned}
\left\|T_{n} x\right\| & \geqq 2^{-n}\left\|T_{n} x_{n}\right\|-\sum_{k=1}^{n-1} 2^{-k}\left\|T_{n} x_{k}\right\|-\sum_{k=n+1}^{\infty} 2^{-k}\left\|T_{n} x_{k}\right\| \\
& \geqq 2^{-n}\left[2^{n}\left(n+1+\sum_{k=1}^{n-1} 2^{-k} M\left(x_{k}\right)\right)\right]-\sum_{k=1}^{n-1} 2^{-k} M\left(x_{k}\right)-\sum_{k=n+1}^{\infty} 2^{-k},
\end{aligned}
$$

since $1 \leqq k \leqq n-1 \Rightarrow\left\|T_{n} x_{k}\right\| \leqq M\left(x_{k}\right)$ and $k \geqq n+1 \Rightarrow\left\|T_{n} x_{k}\right\| \leqq 1$. So, $\left\|T_{n} x\right\| \geqq n+1-\sum_{k=n+1}^{\infty} 2^{-k}>n$, contradicting the pointwise-boundedness of $\mathcal{F}$. Q.E.D.

The Banach-Steinhaus Theorem occurs when $S_{\alpha}=X, \forall \alpha \in A$, and the Pták Theorem when each $T_{\alpha}$ has closed null-space $N\left(T_{\alpha}\right)$, and $S_{\alpha}=N\left(T_{\alpha}\right), \forall \alpha \in A$.

The second theorem concerns mappings into a space with a continuous bilinear form, and is proved in a similar fashion.

DEFinition. A relation $x \perp y$ on a normed linear space is called an orthogonality relation if $x \perp y$ and $x \perp z \Rightarrow x \perp y+z, x \perp y$ and $\alpha$ a scalar $\Rightarrow x \perp \alpha y$, and $x \perp x_{n}$ for $n=1,2, \cdots ; x_{n} \rightarrow x_{0} \Rightarrow x \perp x_{0}$. Given an orthogonality relation $\perp$ on a normed linear space $X$, and given also $x \in X$, we define $S^{\perp}(x)$ to be the closed linear subspace $\{y \in X \mid x \perp y\}$.

THEOREM 2. Let $X$ be a Banach space, $Y, Z$ normed linear spaces and let $\mathfrak{F}=\left\{T_{\alpha} \mid \alpha \in A\right\}$ be a pointwise-bounded family of linear transformations from $X$ to $Y$. Let $\perp$ be an orthogonality relation on $X$, and let $q: Y \times Y \rightarrow Z$ be a continuous bilinear (or conjugate-linear) form. Assume that for each $(\alpha, x) \in A \times X$, the maps $y \rightarrow q\left(T_{\alpha} x, T_{\alpha} y\right)$ is continuous on $S^{\perp}(x)$. Then $\exists x_{1}, \cdots, x_{n} \in X$ such that $1 \leqq i \leqq j \leqq n \Rightarrow x_{i} \perp x_{j}$ and $\exists M>0$ such that $\alpha \in A, x \in \bigcap_{k=1}^{n} S^{\perp}\left(x_{k}\right) \Rightarrow\left\|q\left(T_{\alpha} x, T_{\alpha} x\right)\right\| \leqq M\|x\| .\left\|T_{\alpha} x\right\|$.

Proof. Assume the theorem is false. We assert that, given a finite set $x_{1}, \cdots, x_{n} \in X$ with $x_{i} \perp x_{j}$ for $1 \leqq i \leqq j \leqq n, M>0$, and a finite set $T_{1}, \cdots, T_{n} \in \mathcal{F}$, there is an $x \in X$ and $T \in \mathcal{F}$ with $x_{i} \perp x(1 \leqq i \leqq n)$, $\|x\| \leqq 1,\left\|q\left(T_{k} x_{k}, T_{k} x\right)\right\| \leqq\left\|T_{k} x_{k}\right\|(1 \leqq k \leqq n)$, and $\|q(T x, T x)\|>M\|T x\|$. For $y \in X$, define $M(y)=\sup \left\{\left\|T_{\alpha} y\right\| \mid \alpha \in A\right\}$, and for $(x, \alpha) \in X \times A$, define 


$$
r(x, \alpha)=\inf \left\{\delta \mid y \in S^{\perp}(x) \Rightarrow\left\|q\left(T_{\alpha} x, T_{\alpha} y\right)\right\| \leq \delta\left\|T_{\alpha} x\right\|\|y\|\right\} .
$$

We can assume that, for $1 \leqq k \leqq n, r\left(x_{k}, k\right) \neq 0$, for otherwise the requirement that $\left\|q\left(T_{k} x_{k}, T_{k} x\right)\right\| \leqq\left\|T_{k} x_{k}\right\|$ is trivially satisfied. By assumption, we can find $y \in X$ with $x_{k} \perp y(1 \leqq k \leqq n)$ and $T \in \mathcal{F}$ with $\|q(T y, T y)\|>\gamma M\|y\|\|T y\|$, where $\gamma=\max \left(r\left(x_{1}, 1\right), \cdots, r\left(x_{n}, n\right), 1\right)$. Let $x=(\gamma\|y\|)^{-1} y$, then clearly $\|x\| \leqq 1$. We also have

$$
\begin{aligned}
\|q(T x, T x)\| & =(\gamma\|y\|)^{-2}\|q(T y, T y)\|>(\gamma\|y\|)^{-2} \gamma M\|T y\|\|y\| \\
& =M(\gamma\|y\|)^{-1}\|T y\|=M\|T x\|
\end{aligned}
$$

and, for $1 \leqq k \leqq n$,

$$
\begin{aligned}
\left\|q\left(T_{k} x_{k}, T_{k} x\right)\right\| & =(\gamma\|y\|)^{-1}\left\|q\left(T_{k} x_{k}, T_{k} y\right)\right\| \\
& \leqq\left(\gamma^{-1} r\left(x_{k}, k\right)\right)\|y\|-1\left\|T_{k} x_{k}\right\|\|y\| \leqq\left\|T_{k} x_{k}\right\| .
\end{aligned}
$$

Assuming the theorem false, choose $x_{1} \in X, T_{1} \in \mathcal{F}$ with $\left\|x_{1}\right\| \leqq 1$, $\left\|q\left(T_{1} x_{1}, T_{1} x_{1}\right)\right\|>4\left\|T_{1} x_{1}\right\|$. Having chosen $x_{1}, \cdots, x_{n-1}$ and $T_{1}, \cdots$, $T_{n-1}$, choose $x_{n} \in X, T_{n} \in \mathcal{F}$ with $\left\|x_{n}\right\| \leqq 1, x_{i} \perp x_{n},\left\|q\left(T_{i} x_{i}, T_{i} x_{n}\right)\right\|$ $<\left\|T_{i} x_{i}\right\|$ for $1 \leqq i \leqq n-1$ and

$$
\left\|q\left(T_{n} x_{n}, T_{n} x_{n}\right)\right\|>2^{n}\left(n+1+\sum_{k=1}^{n-1} 2^{-k} M\left(x_{k}\right)\right)\left\|T_{n} x_{n}\right\| .
$$

Let $x=\sum_{k=1}^{\infty} 2^{-k} x_{k}$, by completeness, $x \in X$. We can assume without loss of generality that $y, z \in Y \Rightarrow\|q(y, z)\| \leqq\|y\|\|z\|$. Then for each integer $n$,

$$
\begin{aligned}
\left\|T_{n} x\right\|\left\|T_{n} x_{n}\right\| \geqq\left\|q\left(T_{n} x_{n}, T_{n} x\right)\right\| & =\left\|q\left(T_{n} x_{n}, T_{n}\left(\sum_{k=1}^{\infty} 2^{-k} x_{k}\right)\right)\right\| \\
= & \| 2^{-n} q\left(T_{n} x_{n}, T_{n} x_{n}\right)+\sum_{j=1}^{n-1} 2^{-j} q\left(T_{n} x_{n}, T_{n} x_{j}\right) \\
& +q\left(T_{n} x_{n}, T_{n}\left(\sum_{k=n+1}^{\infty} 2^{-k} x_{k}\right)\right) \| .
\end{aligned}
$$

Since $S^{\perp}\left(X_{n}\right)$ is a closed subspace, $k \geqq n+1 \Rightarrow x_{k} \in S^{\perp}\left(x_{n}\right)$, and $y \rightarrow q\left(T_{n} x_{n}, T_{n} y\right)$ is continuous on $S^{\perp}\left(x_{n}\right)$, so we see that

$$
q\left(T_{n} x_{n}, T_{n}\left(\sum_{k=n+1}^{\infty} 2^{-k} x_{k}\right)\right)=\sum_{k=n+1}^{\infty} 2^{-k} q\left(T_{n} x_{n}, T_{n} x_{k}\right) .
$$

Now $1 \leqq k \leqq n-1 \Rightarrow\left\|q\left(T_{n} x_{n}, T_{n} x_{k}\right)\right\| \leqq\left\|T_{n} x_{n}\right\|\left\|T_{n} x_{k}\right\| \leqq M\left(x_{k}\right)\left\|T_{n} x_{n}\right\|$ and $k \geqq n+1 \Longrightarrow\left\|q\left(T_{n} x_{n}, T_{n} x_{k}\right)\right\| \leqq\left\|T_{n} x_{n}\right\|$. So 


$$
\begin{aligned}
\left\|T_{n} x\right\|\left\|T_{n} x_{n}\right\| \geqq & 2^{--n}\left\|q\left(T_{n} x_{n}, T_{n} x_{n}\right)\right\|-\sum_{k=1}^{n-1} 2^{-k}\left\|q\left(T_{n} x_{n}, T_{n} x_{k}\right)\right\| \\
& -\sum_{k=n+1}^{\infty} 2^{-k}\left\|q\left(T_{n} x_{n}, T_{n} x_{k}\right)\right\| \\
> & 2^{-n}\left[2^{n}\left(n+1+\sum_{k=1}^{n-1} 2^{-k} M\left(x_{k}\right)\right)\right]\left\|T_{n} x_{n}\right\| \\
& -\sum_{k=1}^{n-1} 2^{-k}\left\|T_{n} x_{n}\right\| M\left(x_{k}\right)-\sum_{k=n+1}^{\infty} 2^{-k}\left\|T_{n} x_{n}\right\| \\
= & \left(n+1-\sum_{k=n+1}^{\infty} 2^{-k}\right)\left\|T_{n} x_{n}\right\|>n\left\|T_{n} x_{n}\right\|
\end{aligned}
$$

since $T_{n} x_{n} \neq 0$ we have $\left\|T_{n} x\right\|>n$, contradicting the pointwiseboundedness of $\mathcal{F}$. Q.E.D.

Although the Banach-Steinhaus Theorem and its uses are well known, the second theorem is a bit more obscure. The following is an example of its utility. Let $H, K$ be Hilbert spaces, $T: H \rightarrow K$ a linear map such that $(x, y)=0$ in $H \Rightarrow|(T x, T y)| \leqq C\|x\|\|y\|$. Then $T$ is continuous. Theorem 2 yields the existence of $x_{1}, \cdots, x_{n}$ such that $\left(x, x_{k}\right)=0$ for $1 \leqq k \leqq n \Rightarrow|(T x, T x)| \leqq M\|T x\|\|x\|$, and so $\|T x\|^{2}$ $\leqq M\|T x\|\|x\| \Rightarrow\|T x\| \leqq M\|x\|$. Letting $B$ denote the closed subspace spanned by $x_{1}, \cdots, x_{n}$, we have $H=B \oplus B^{\perp}$, and $T \mid B^{\perp}$ is continuous. But $B$ is finite dimensional, and so $T \mid B$ is continuous, completing the proof.

These techniques can also be applied to homomorphisms of Banach algebras. One can modify the Main Boundedness Theorem of Badé and Curtis to obtain

TheOREM 3. Let $\left\{T_{\alpha} \mid \alpha \in A\right\}$ be a pointwise-bounded family of homomorphisms of a Banach algebra $X$ into a Banach algebra $Y$. Let $\left\{x_{n} \mid n=1,2, \cdots\right\},\left\{y_{n} \mid n=1,2, \cdots\right\}$ be sequences from $X$ such that $x_{n} y_{m}=0$ for $n \neq m$. Then

$$
\sup \left\{\left\|T_{\alpha}\left(x_{n} y_{n}\right)\right\| /\left\|x_{n}\right\|\left\|y_{n}\right\| \mid \alpha \in A, n=1,2, \cdots\right\}<\infty .
$$

Here we have used the formulation of the Main Boundedness Theorem given in [1]. Techniques similar to those used in Theorem 1 will show that the theorems of [2] and [5] which relate to continuity of a homomorphism also hold for equicontinuity of a pointwisebounded family of homomorphisms. For example, Theorem II.1 of [5] can be changed to show that every pointwise-bounded family of 
Banach algebra homomorphisms of a von Neumann algebra is equicontinuous on a dense subalgebra.

\section{BibLIOGRAPHY}

1. G. Bachelis, Homomorphisms of annihilator Banach algebras. II, Pacific J. Math. 30 (1969), 283-292.

2. W. Badé and P. Curtis, Homomorphisms of commutative Banach algebras, Amer. J. Math. 82 (1960), 589-608. MR 22 \#8354.

3. N. Dunford and J. Schwartz, Linear operators. I: General theory, Pure and Appl. Math., vol. 7, Interscience, New York, 1958. MR 22 \#8302.

4. V. Pták, $A$ uniform boundedness theorem and mappings into spaces of operators, Studia Math. 31 (1968), 425-431. MR 38 \#4967.

5. J. Stein, Continuity of homomorphisms of von Neumann algebras, Amer. J. Math. 91 (1969), 153-159. MR 39 \#6095.

University of California, Los Angeles, California 90024

Aarhus University, Aarhus, Denmark 